\title{
Super-resolution imaging and performance optimization for single- and multi-layer silver superlenses
}

\author{
R. J. Blaikie and D. O. S. Melville \\ MacDiarmid Institute for Advanced Materials and Nanotechnology, Department of Electrical and Computer Engineering, University of \\ Canterbury, Private Bag 4800, Christchurch, New Zealand \\ r.blaikie@elec.canterbury.ac.nz
}

\begin{abstract}
Super-resolution imaging has been achieved in a lithography environment using both single- and multi-layer silver superlenses. The performance of these systems is compared here, and analytical and simulation methods are used to optimize performance.

(C)2006 Optical Society of America

OCIS codes: (240.6680) Surface plasmons; (110.5220) Photolithography
\end{abstract}

\section{Introduction}

For conventional optical imaging and projection lithography the resolution is limited to approximately half the exposing wavelength. The trend in semiconductor manufacturing has therefore been to use shorter wavelengths, with $193 \mathrm{~nm}$ ArF laser sources being used currently. Another way to improve resolution is to go into the near field region, where conventional resolution limits no longer apply. We have used our Evanescent Near Field Optical Lithography (ENFOL) technique to demonstrate resolution down to $\lambda / 7$ in a hard-contact lithography experiment [1], with $\lambda / 20$ resolution predicted for thinner resists [2].

A problem with ENFOL and related techniques is that they require intimate mask/resist contact, which may be undesirable in a manufacturing environment. Following Pendry's proposal that a planar metal film can act as a plasmon-mediated near-field superlens [3] a near-field planar lens lithography (PLL) technique has been developed in which a planar metallic film is inserted between the mask and imaging photoresist [4]. Using PLL we have provided the first experimental demonstration of sub-wavelength imaging using a silver planar lens illuminated at the i-line wavelength of a Mercury lamp [5]. We have gone on to show that this technique is capable of producing sub-diffraction-limited near-field images [6], as have another group using a related technique [7].

Further improvements in the imaging properties of silver superlenses have been proposed by going from singlelayer to multi-layer lens structures [8]. If the same total thickness of silver and dielectric spacer layers is used, then the lamination of these materials provides resolution enhancements by the introduction of additional metal surfaces between the object and image planes; as the imaging in these silver superlenses is strongly mediated by surface plasmons, the incorporation of additional layers on which such plasmons can be generated allows higher spatial frequency components to be efficiently transferred through the system.

The resolution enhancements provided in going to multi-layer superlenses have been experimentally tested very recently [9]. This work showed that super-resolution can also be achieved through a double-layer silver superlens, with enhanced transmission compared to a single-layer lens. We review this work here and present the main experimental findings. Analytical and simulation results are then given to show how the performance of multi-layer superlenses can be optimized by changing the relative layer thicknesses in the lens stack.

\section{Experimental Methods}

ENFOL and PLL are based on conformal-mask contact lithography [10], and the experimental arrangements are shown schematically in Fig. 1. In both cases a 30-nm thick tungsten mask is patterned on a thin glass substrate (either 100 or $150 \mu \mathrm{m}$ thick) using electron-beam lithography and reactive ion etching. For ENFOL this conformal mask is then mounted on a rigid support and used in a commercial mask aligner (Karl Suss, MA-6). For PLL the mask is coated with a planarized poly-methylmethacrylate (PMMA) dielectric spacer, a silver (Ag) lensing layer and a silicon dioxide $\left(\mathrm{SiO}_{2}\right)$ dielectric spacer prior to being mounted on its rigid support. For multi-layer lenses the deposition of alternating layers of $\mathrm{Ag}$ and $\mathrm{SiO}_{2}$ is repeated until the final lens stack is complete. Full details of the experimental procedures have been published previously $[5,6]$, and the additional surface roughness that is introduced when going to multi-layer lenses is characterized in Ref. 9. A shorthand notation has also been used for describing the lens stacks, in which a system with $25 \mathrm{~nm}$ of PMMA, $50 \mathrm{~nm}$ of Ag, followed by $10 \mathrm{~nm}$ of $\mathrm{SiO}_{2}$ is referred to as a $25 / 50 / 10-\mathrm{PMMA} / \mathrm{Ag} / \mathrm{SiO}_{2}$ lens; the notation extends naturally to multi-layer systems. 

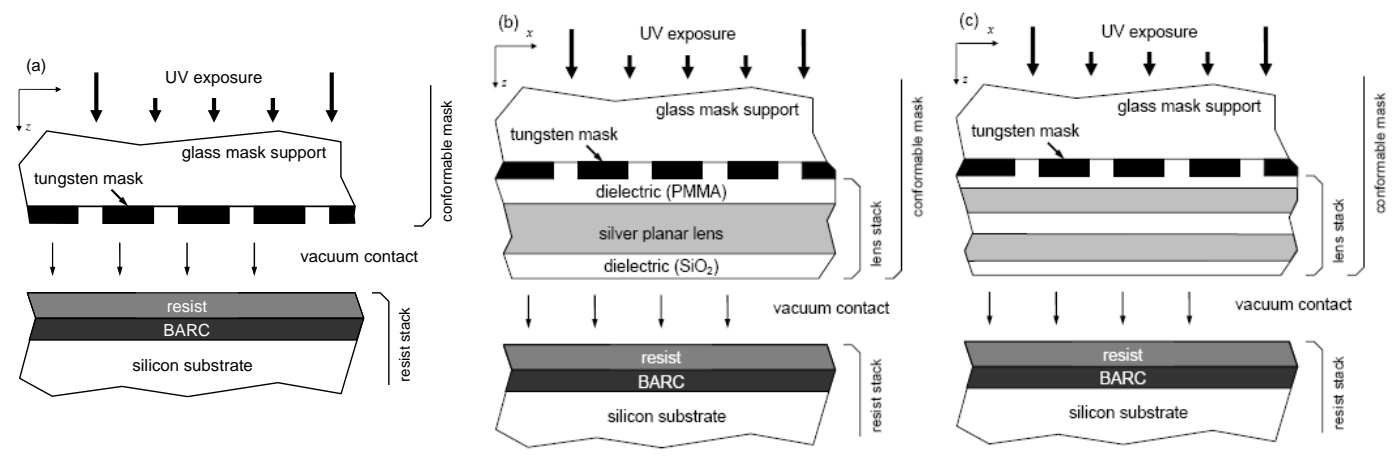

Fig. 1. Schematic diagrams of the experimental configurations for (a) the ENFOL technique, (b) single-layer planar lens lithography (PLL), and (c) multi-layer PLL. Photoresist and Bottom Anti-Reflection Coating (BARC) layers on silicon are used to capture images prior to Atomic Force Microscope (AFM) analysis.

\section{Experimental Results}

A number of different silver-layer thicknesses have been employed in PLL imaging experiments to date, from $120 \mathrm{~nm}$ down to below $50 \mathrm{~nm}$, with the image resolution improving as the silver thickness is reduced, as expected from work that has shown that optimal evanescent-field transmission occurs for silver layers around 35-nm thick [11]. One interesting point to note here is that the effects of surface roughness on the silver film in acting as a local source of surface plasmons can often be clearly seen in the results of PLL exposures, as shown in Fig. 2 for a $1-\mu \mathrm{m}$ period grating imaged through a 40/85/26-PMMA/Ag/ $\mathrm{SiO}_{2}$ lens stack; here there a number of dark spots on the image, surrounded by weak concentric rings. We attribute these to exposure from high intensity 'hot spots' on the silver layer, where the surface roughness acts as a strong source of surface plasmons that are capable of exposing the resist, which is expected if the conditions for super-resolution imaging are to be met $[4,5]$.

Comparisons between images formed using single-layer and double-layer lenses (Fig. 2(b)) show that subdiffraction-limited resolution can be achieved in both cases, but that the image fidelity is degraded for 170-nm pitch features due to additional surface-roughness effects [9]. One positive feature that has been experimentally determined for the double-layer lenses is that the required exposure time reduces by approximately a factor of two for the same total silver thickness, which is evidence that the transmission is enhanced by laminating the silver.

(a)

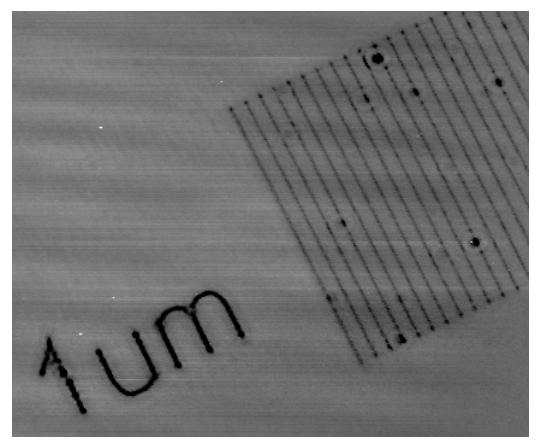

(b)

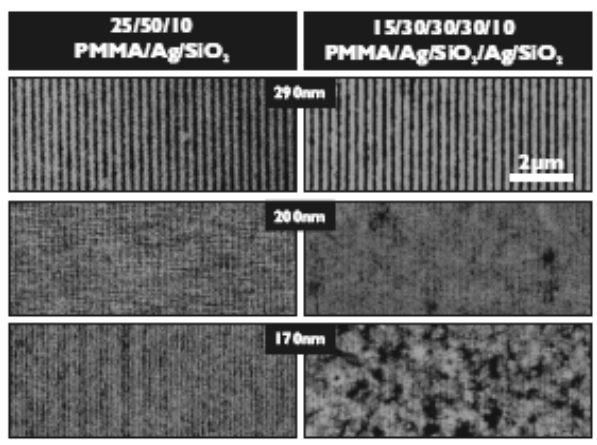

Fig. 2. (a) AFM scan of a $1-\mu \mathrm{m}$ period grating imaged through a 40/85/26-PMMA/Ag/ $/ \mathrm{SiO}_{2}$ lens (height scale is $40 \mathrm{~nm}$ ). (b) AFM scans comparing single-layer 25/50/10-PMMA/Ag/SiO2 and a double-layer 15/30/30/30/10-PMMA/Ag/SiO2/Ag/SiO2 lenses. All height scales are 50nm. (From Ref. 9)

\section{Simulations and System Optimization}

Finite-difference time-domain (FDTD) simulations have been performed for the PLL geometries that have been studied experimentally, and examples of the expected intensity profiles in the imaging resist are shown in Fig. 3(a) for three cases: proximity lithography with an $85 \mathrm{~nm}$ gap and the two PLL geometries used for the images in Fig. 2(b). For the 170-nm period structures used in this simulation the ability of the silver superlenses to project high-contrast near field images is apparent (noting also that there is only a weak, inverted image for the proximity exposure), and the experimentally-observed intensity enhancement for the double-layer lens is also clear. Future challenges in the FDTD simulation work lie in the ability to model the effects of surface roughness on the imaging. 

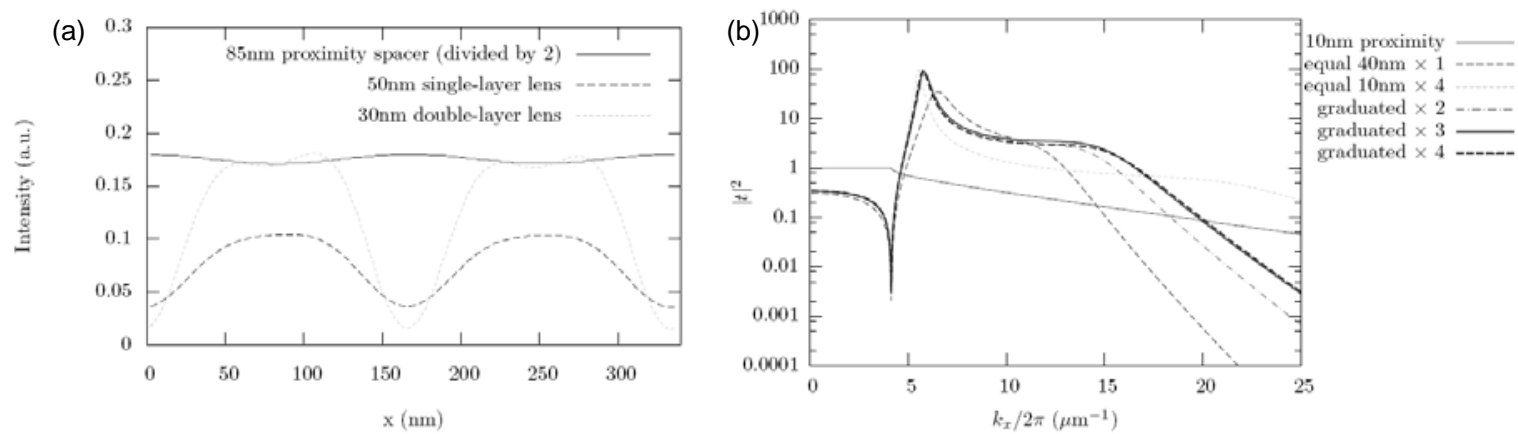

Fig. 3. (a) Simulated intensity profiles for the single- and double-layer lens exposures of Fig. 2(b), compared to a proximity exposure. (b) Transmission coefficients (taken $10 \mathrm{~nm}$ into the resist) versus spatial frequency for various PLL lens stacks, with 10 -nm proximity exposure shown for example.

An analytical approach has also been used to investigate the optimization of multi-layer lenses [12]. An example of the differences in the spatial-frequency transfer functions for various lens stacks is shown in Fig. 3(b), with the details of the layer thicknesses given in Table 1; in each case the total silver thickness is $40 \mathrm{~nm}$ with the image plane taken $10 \mathrm{~nm}$ below the final silver layer, and the results are compared to a simple $10 \mathrm{~nm}$ proximity exposure. Enhancements in the super-resolved spatial frequency components (those above $5 \mu \mathrm{m}^{-1}$ ) are observed for all the superlens geometries, and the equi-spaced four-layer lens has the highest bandwidth with useful performance out to 5 times the diffraction limit $\left(25 \mu \mathrm{m}^{-1}\right)$. However, by changing the relative thickness of the layers the performance can be optimized for certain spatial frequencies. For example, 3- or 4-layer graduated lenses show intensity enhancements of greater than 100 times at $6 \mu \mathrm{m}^{-1}$, with better performance than the equi-spaced lens out to $20 \mu \mathrm{m}^{-1}$. The differences between the range of graduation schemes studied is rather subtle, indicating that good performance can be achieved for a range of multi-layer lens geometries, with the corollary that the practical performance of any particular superlensing system will not be extremely sensitive to the exact dimensions of the layers used.

Table 1. Layer thicknesses for the various multi-layer lens stacks analyzed. The silver layers are in bold text.

\begin{tabular}{c|cccccccc}
\hline Lens stack & \multicolumn{8}{|c}{ Dimensions $\mathbf{( n m )}$} \\
\hline equal $10 \mathrm{~nm} \times 4$ & 5 & $\mathbf{1 0}$ & 5 & $\mathbf{1 0}$ & 5 & $\mathbf{1 0}$ & 5 & $\mathbf{1 0}$ \\
\hline graduated $\times 2$ & 5 & $\mathbf{1 0}$ & 20 & $\mathbf{3 0}$ & & & & \\
\hline graduated $\times 3$ & 1.5 & $\mathbf{3 . 1}$ & 6.2 & $\mathbf{9 . 2}$ & 16.9 & $\mathbf{2 7 . 7}$ & & \\
\hline graduated $\times 4$ & 0.5 & $\mathbf{1}$ & 2 & $\mathbf{3}$ & 5.5 & $\mathbf{9}$ & 18 & $\mathbf{2 7}$ \\
\hline
\end{tabular}

The effects that have been predicted here will be challenging to test experimentally, but the prospect of resolving 20-nm half-pitch features ( $25 \mu \mathrm{m}^{-1}$ spatial features) using a conventional UV exposure tool is an extremely enticing one. As the experimental aspects of this study have already shown, it is control over the surface roughness, rather than the absolute layer thicknesses, that will be critical in this endeavor.

\section{References}

[1] M. M. Alkaisi, R. J. Blaikie, S. J. McNab, R. Cheung and D. R. S. Cumming, "Sub-diffraction-limited patterning using evanescent nearfield optical lithography," Appl. Phys. Lett. 75, 3560-3562 (1999).

[2] S. J. McNab and R. J. Blaikie, "Contrast in the evanescent near field of $\lambda / 20$ period gratings for photolithography," Appl. Opt. 39, 20-25 (2000).

[3] J. B. Pendry, "Negative refraction makes a perfect lens," Phys. Rev. Lett. 85, 3966-3969 (2000).

[4] R. J. Blaikie and S. J. McNab, "Simulation study of "perfect lenses' for near-field optical nanolithography," Microelectron. Eng. 61-2, 97-103 (2002).

[5] D. O. S. Melville, R. J. Blaikie and C. R. Wolf, "Submicron imaging with a planar silver lens," Appl. Phys. Lett. 84, 4403-4405 (2004).

[6] D. O. S. Melville and R. J. Blaikie "Super-resolution imaging through a planar silver layer," Opt. Express 13, 2127-2134 (2005).

[7] N. Fang, H. Lee, C. Sun and X. Zhang, "Sub-diffraction-limited imaging with a silver superlens," Science 308, $534-537$ (2005).

[8] S. A. Ramakrishna "Physics of negative refractive index materials," Rep. Prog. Phys. 68, 448-521 (2005).

[9] D. O. S. Melville and R. J. Blaikie, "Experimental comparison of resolution and pattern fidelity in single- and double-layer planar lens lithography," J. Opt. Soc. Am. B 23, vol. 3, in press (2006).

[10] H. I. Smith, "Fabrication techniques for surface-acoustic-wave and thin-film optical devices," Proc. IEEE 62, 1361-1387 (1974).

[11] Z. W. Liu, N. Fang, T. J. Yen and X. Zhang, "Rapid growth of evanescent waves by a silver superlens," Appl. Phys. Lett. 83, 5184-5186 (2003).

[12] D. O. S. Melville "Planar lensing lithography: enhancing the near field," PhD thesis, University of Canterbury, New Zealand (2006). 